\title{
Predictors of Reintubation in Critically Ill Patients
}

\author{
Timothy Miu MD, Aaron M Joffe DO, N David Yanez PhD, Nita Khandelwal MD, \\ Armagan HC Dagal MD FCRA, Steven Deem MD, and Miriam M Treggiari MD PhD MPH
}

\begin{abstract}
BACKGROUND: Assessment of a patient's readiness for removal of the endotracheal tube in the ICU is based on respiratory, airway, and neurological measures. However, nearly $20 \%$ of patients require reintubation. We created a prediction model for the need for reintubation, which incorporates variables importantly contributing to extubation failure. METHODS: This was a cohort study of 2,007 endotracheally intubated subjects who required ICU admission at a tertiary care center. Data collection included demographic, hemodynamic, respiratory, and neurological variables preceding extubation. Data were compared between subjects extubated successfully and those who required reintubation, using bivariate logistic regression models, with the binary outcome reintubation and the baseline characteristics as predictors. Multivariable logistic regression analysis with robust variance was used to build the prediction model. RESULTS: Of the 2,007 subjects analyzed, $376(19 \%)$ required reintubation. In the bivariate analysis, admission Simplified Acute Physiology Score II, minute ventilation, breathing frequency, oxygenation, number of prior SBTs, rapid shallow breathing index, airway-secretions suctioning frequency and quantity, heart rate, and diastolic blood pressure differed significantly between the extubation success and failure groups. In the multivariable analysis, higher Simplified Acute Physiology Score II and suctioning frequency were associated with failed extubation. The area under the receiver operating characteristic curve was 0.68 for failure at any time, and $\mathbf{0 . 7 1}$ for failure within 24 hours. However, prior failed SBT, minute ventilation, and diastolic blood pressure were additional independent predictors of failure at any time, whereas oxygenation predicted extubation failure within 24 hours. CONCLUSIONS: A small number of independent variables explains a substantial portion of the variability of extubation failure, and can help identify patients at high risk of needing reintubation. These characteristics should be incorporated in the decision-making process of ICU extubation. Key words: extubation; mechanical ventilation; ICU; cohort study; airway management; clinical prediction model; human. [Respir Care 2014;59(2):178-185. (C) 2014 Daedalus Enterprises]
\end{abstract}

\section{Introduction}

The decision to liberate a patient from invasive mechanical ventilation is a 2 -component process that requires cli-

\footnotetext{
The authors are affiliated with the Department of Anesthesiology and Pain Medicine, Harborview Medical Center, with the exception of Dr Yanez, who is affiliated with the Department of Biostatistics, School of Public Health and Community Medicine, School of Medicine, University of Washington, Seattle, Washington. Dr Treggiari is also affiliated with the Department of Epidemiology, School of Public Health and Community Medicine, University of Washington, Seattle, Washington. Dr Deem is also affiliated with the Division of Pulmonary and Critical Care Medicine, Department of Medicine, University of Washington, Seattle, Washington.
}

nicians to assess the continued need for mechanical ventilation and the need for the artificial airway. Typically,

\footnotetext{
This study was partly supported by a grant from Cook Medical Group. The authors have disclosed no conflicts of interest.

Dr Miu presented a version of this paper at the annual meeting of the American Society of Anesthesiologists, held October 13-17, 2012, in Washington DC.

Correspondence: Miriam M Treggiari MD PhD MPH, Department of Anesthesiology and Pain Medicine, Box 359724, Harborview Medical Center, University of Washington School of Medicine, 325 Ninth Avenue, Seattle, WA 98104. E mail: treggmm@u.washington.edu.
}

DOI: $10.4187 /$ respcare. 02527 
assessment of gas exchange and pulmonary mechanics occurs with the performance of a spontaneous breathing trial (SBT). ${ }^{1}$ Single-point-in-time examination of the ratio of respiratory frequency to tidal volume (the rapid shallow breathing index), integration of thoracic compliance, breathing frequency, arterial oxygenation, and maximum occlusion pressure, or dynamic changes in these indices over the course of the SBT differentiate between successes and failures of this trial. ${ }^{2-4}$ Nonetheless, even after passing an SBT, nearly one in 5 will require reintubation at some time during the hospital stay, with half of these patients requiring reintubation within the first 24 hours. ${ }^{3,5}$ Salam et $\mathrm{al}^{6}$ reported the ability to maintain a patent upper airway and clear secretions, indicated by a cough peak

\section{See the Related Editorial on Page 301}

flow $>60 \mathrm{~L} / \mathrm{min}$, tracheal secretions $<2.5 \mathrm{~mL} / \mathrm{min}$, and the ability to follow 4 simple tasks (ie, open eyes, track with eyes, grasp with hands, and stick out tongue) increased the odds of successful extubation. Mokhlesi and colleagues $^{7}$ reported on 122 patients who were followed after extubation to identify those who were reintubated within 48 hours. Of the $13 \%$ of patients who required early reintubation, moderate to copious tracheal secretions, Glasgow coma score $<10$, and a $\mathrm{P}_{\mathrm{aCO}_{2}}>44 \mathrm{~mm} \mathrm{Hg}$ during the SBT all independently predicted failure. Unfortunately, the ability to generalize these results to larger and more diverse patient groups is limited by the small numbers of observations included in these reports. Despite a wealth of literature reporting the associations between a variety of individual indices and success or failure of extubation, few studies have reported on clinical decision rules that could better predict what patients are at risk for extubation failure after the successful completion of a SBT.

Thus, the aim of the present study was to build a model with high precision to predict extubation failure early (reintubation within the first $24 \mathrm{~h}$ ) or at any time during the hospitalization, using commonly available bedside preextubation variables, in a large population of critically ill adults.

\section{Methods}

\section{Study Setting}

The University of Washington institutional review board approved this study with a waiver of informed consent. The study site was Harborview Medical Center, a 413-bed level 1 trauma hospital in Seattle, Washington, affiliated with the University of Washington, and serving as a tertiary referral center for critically ill patients in a 4-state region (Washington, Alaska, Montana, and Idaho). There

\section{QUICK LOOK}

\section{Current knowledge}

Reintubation is common following extubation and discontinuation of mechanical ventilation. The extubation failure rate considered acceptable has empirically been set at $10-15 \%$, but avoiding reintubation remains an important clinical goal.

\section{What this paper contributes to our knowledge}

Extubation failure occurred in 19\% of subjects. Risk factors for reintubation included disease severity, secretions burden, and minute ventilation $>8 \mathrm{~L} / \mathrm{min}$. Patients with lower $\mathrm{P}_{\mathrm{aO}} / \mathrm{F}_{\mathrm{IO}_{2}}$ and those who had undergone more spontaneous breathing trials prior to extubation were at higher risk for extubation failure in the first 24 hours.

are 88 ICU beds, distributed among medical/cardiac (18 beds), trauma/surgical ( 24 beds), cardiac, neurology/ neurosurgical ICUs ( 28 beds), and pediatric/burn ICU (18 beds). The ICUs admit approximately 3,500 patients per year, and over half of them are mechanically ventilated for any duration of time during their hospital stay. These ICUs are covered 24 hours a day by an intensivist-led team consisting of an attending physician, critical care fellow, senior resident, and junior resident. The departments of surgery, anesthesiology, internal medicine, neurology, and emergency medicine provide physician coverage, attendings, and trainees. A dedicated ICU team rounds at least once a day and writes orders. Respiratory therapists help to manage all ventilated patients, including performance of spontaneous breathing trials (SBTs). Elements of airway assessment and management are done by the ICU team.

\section{Study Eligibility Criteria}

All patients who were admitted to one of the ICUs and required invasive mechanical ventilation via an endotracheal tube placed in the pre-hospital or hospital setting, in the period between July 1, 2008, and August 31, 2009, were eligible for inclusion. Patients less than 18 years of age and those extubated in the context of comfort care, after tracheostomy placement, or who died prior to extubation were excluded. Patients who had airway management for elective or emergency procedures in the operating room were not considered as ICU extubation failures.

All subjects underwent a daily protocolized ventilator weaning assessment as follows. Subjects were considered for an SBT if they met the following criteria: evidence of resolution or improvement of the underlying cause 
of respiratory failure, minute ventilation $<15 \mathrm{~L} / \mathrm{min}$, PEEP $\leq 8 \mathrm{~cm} \mathrm{H} \mathrm{H}_{2}, \mathrm{~F}_{\mathrm{IO}_{2}}<0.5, \mathrm{P}_{\mathrm{aO}_{2}} / \mathrm{F}_{\mathrm{IO}_{2}} \geq 150 \mathrm{~mm} \mathrm{Hg}$, $\mathrm{pH} \geq 7.25$, intact respiratory drive, intracranial pressure $<20 \mathrm{~cm} \mathrm{H}_{2} \mathrm{O}$, and hemodynamic stability without cardiovascular support. Sedation for mechanical ventilation was provided per standardized ICU protocol, targeting a goal of 0 to -1 on the Richmond Agitation and Sedation Scale. Unless contraindicated, a spontaneous awakening trial was performed daily, followed by an SBT. ${ }^{8}$ Per routine protocol, all SBTs are performed with a continuous positive pressure of $5 \mathrm{~cm} \mathrm{H}_{2} \mathrm{O}$, without additional pressure support. Failure of the SBT was defined as: breathing frequency $>35$ breaths/min, oxygen saturation $<90 \%$ for $>30$ seconds, reduction in minute ventilation to $<75 \%$ of baseline during mechanical ventilation, heart rate $>140$ beats $/ \mathrm{min}$ or a change of $>20 \%$ from baseline, systolic blood pressure $>180$ or $<90 \mathrm{~mm} \mathrm{Hg}$, sustained increase in anxiety, diaphoresis or other clinical sign of respiratory distress, increase in intracranial pressure $>20 \mathrm{~cm} \mathrm{H}_{2} \mathrm{O}$ for $>2 \mathrm{~min}$, arrhythmia, $\mathrm{pH} \leq 7.25$, or $\mathrm{P}_{\mathrm{aCO}_{2}}$ increase of $\geq 10 \mathrm{~mm} \mathrm{Hg} .{ }^{9}$ Additional factors considered prior to extubation, after the subject has passed an SBT, included the requirement for tracheal suctioning more than every 4 hours, presence of an effective spontaneous cough, presence of a leak around the deflated tracheal tube cuff with a sustained manual inspiratory pressure of $<30 \mathrm{~cm} \mathrm{H}_{2} \mathrm{O}$, and absence of airway reflexes. These criteria applied to all intubated patients through a hospital protocol, unless extubation was unplanned.

\section{Data Collection}

Data were abstracted via automated search from the electronic medical record. Manual review of the electronic medical record was conducted as needed for variables not retrievable through the automated search and for data verification. Demographic variables collected included age, sex, ethnicity, height, weight, hospital and ICU stay, and severity of illness, measured with the Simplified Acute Physiology Score II (SAPS II). Admission services included medical, general surgery, neurological surgery, orthopedic surgery, vascular surgery, and otolaryngology.

Clinical variables that were recorded included ventilatory variables $\left(\mathrm{F}_{\mathrm{IO}_{2}}\right.$, PEEP, amount of pressure support, tidal volume, minute ventilation, and breathing frequency); the number of attempted SBTs prior to actual extubation; arterial blood gas values at the end of the SBT $(\mathrm{pH}$, $\mathrm{P}_{\mathrm{aCO}_{2}}$, and $\mathrm{P}_{\mathrm{aO}_{2}}$ ); cumulative 24-hour suctioning frequency and quantity of secretions (graded as copious [3 points], moderate [ 2 points], or mild [ 1 point]); the need for oral suctioning; and the presence or absence of endotracheal tube cuff leak. Hemodynamic and continuously monitored variables included systolic, mean, and diastolic blood pressure, heart rate, and $\mathrm{S}_{\mathrm{pO}_{2}}$. Neurological variables included: Glasgow coma score and the numeric score for each re- sponse (eyes, verbal, motor); presence or absence of a gag reflex; pupillary light reflex; and intracranial pressure, when available. The value that was used in our study was obtained either during the last successful SBT, or during the SBT itself.

Decisions regarding extubation and the need for reintubation, if applicable, were made as part of routine clinical care by the primary ICU service caring for the subject. For subjects requiring reintubation, the time to reintubation in hours was abstracted from the record.

\section{Study End Points and Definitions}

Subjects were classified in 2 groups based on the requirement for reintubation: that is, "never reintubated" versus "one or more reintubations" during the hospital stay, not including intubations for operating room procedures. The primary end point was success of extubation, defined as reaching hospital discharge without requiring out-ofoperating room reintubation. Secondary end points were vital signs, respiratory and ventilator variables, and neurologic exam findings during SBT or immediately prior to extubation.

Extubation failure was defined as the requirement for reinstitution of mechanical ventilation any time after initial extubation, and requiring out-of-operating room tracheal intubation during the hospital stay. Early extubation failure was defined as extubation failure $\leq 24$ hours after extubation. Reintubation events in the operating room for elective or emergency procedures did not count toward the study-defined out-of-operating room reintubation.

\section{Statistical Analysis}

Baseline characteristics were compared between the groups of subjects never reintubated versus those who were reintubated outside the operating room at least once, using bivariate logistic regression models, with the binary outcome reintubation, and the baseline characteristics as predictors. Patterns of missing values were explored in descriptive analyses, and missing data among variables are reported in the tables. For the primary analysis, multivariable logistic regression was used to estimate the adjusted odds ratio of reintubation to account for important predictors, explanatory variables, and potential confounders. We included predictors that are potential confounders and known risk factors: age, sex, body mass index, and SAPS II. Variables thought to be collinear were not included simultaneously in the model if their correlation coefficient was $>0.6$. Robust (sandwich) variance estimates were used in all regression models to compute the confidence intervals. We planned a priori a secondary analysis that was restricted to reintubation events that occurred within 24 hours of extubation. A 2-sided alpha level of .05 was considered 
Table 1. Characteristics Prior to the Last Successful Spontaneous Breathing Trial

\begin{tabular}{|c|c|c|c|c|c|}
\hline & $\begin{array}{l}\text { All Patients } \\
(n=2,007)\end{array}$ & $\begin{array}{l}\text { Not Reintubated } \\
\quad(n=1,628)\end{array}$ & $\begin{array}{l}\text { Reintubated } \\
(n=379)\end{array}$ & AUC* & $P \dagger$ \\
\hline Tidal volume, $\mathrm{mL}$ & $534 \pm 94(1,720)$ & $534 \pm 94(1,400)$ & $525 \pm 98(320)$ & 0.524 & .18 \\
\hline Breathing frequency, breaths/min & $18 \pm 6(1,811)$ & $18 \pm 6(1,466)$ & $20 \pm 6(345)$ & 0.596 & $<.01$ \\
\hline Minute ventilation, $\mathrm{L} / \mathrm{min}$ & $7.6 \pm 2.9(898)$ & $7.4 \pm 2.9(711)$ & $8.5 \pm 2.9(187)$ & 0.615 & $<.01$ \\
\hline PEEP, $\mathrm{cm} \mathrm{H}_{2} \mathrm{O}$ & $5 \pm 1(1,804)$ & $5 \pm 1(1,459)$ & $5 \pm 1(345)$ & 0.503 & .53 \\
\hline $\mathrm{F}_{\mathrm{IO}_{2}}$ & $0.40 \pm 0.10(1,809)$ & $0.41 \pm 0.11(1,464)$ & $0.40 \pm 0.8(345)$ & 0.498 & .18 \\
\hline $\mathrm{S}_{\mathrm{pO}_{2}}, \%$ & $98.8 \pm 1.8(1,813)$ & $98.9 \pm 1.8(1,468)$ & $98.6 \pm 1.9(345)$ & 0.557 & $<.01$ \\
\hline Suctioning frequency, suctionings/24 h & $6.9 \pm 4.2(1,771)$ & $6.6 \pm 4.1(1,428)$ & $8.4 \pm 4.0(343)$ & 0.629 & $<.01$ \\
\hline Secretions quantity score & $9.6 \pm 7.3(1,769)$ & $9.0 \pm 7.0(1,425)$ & $12.2 \pm 8.0(344)$ & 0.633 & $<.01$ \\
\hline Positive cuff leak, no. (\%) & $138(86)$ & $109(86)$ & $29(85)$ & 0.503 & .94 \\
\hline Heart rate, beats/min & $90 \pm 19(1,810)$ & $90 \pm 19(1,465)$ & $92 \pm 18(345)$ & 0.535 & .05 \\
\hline Systolic blood pressure, $\mathrm{mm} \mathrm{Hg}$ & $130 \pm 22(1,810)$ & $130 \pm 22(1,465)$ & $128 \pm 24(345)$ & 0.528 & .23 \\
\hline Diastolic blood pressure, $\mathrm{mm} \mathrm{Hg}$ & $75 \pm 15(1,810)$ & $75 \pm 14(1,465)$ & $72 \pm 16(348)$ & 0.556 & $<.01$ \\
\hline Mean arterial pressure, $\mathrm{mm} \mathrm{Hg}$ & $92 \pm 16(1,789)$ & $93 \pm 16(1,450)$ & $91 \pm 17(342)$ & 0.540 & .06 \\
\hline Glasgow coma score & $9.7 \pm 1.9(1,733)$ & $9.8 \pm 1.9(1,404)$ & $9.6 \pm 1.7(329)$ & 0.521 & .17 \\
\hline Eye & $3.1 \pm 1.0(1,738)$ & $3.1 \pm 1.0(1,408)$ & $3.1 \pm 1.0(330)$ & 0.507 & .71 \\
\hline Verbal & $1.1 \pm 0.7(1,738)$ & $1.2 \pm 0.8(1,408)$ & $1.1 \pm 0.6(330)$ & 0.509 & .13 \\
\hline Motor & $5.6 \pm 0.9(1,735)$ & $5.6 \pm 0.9(1,405)$ & $5.5 \pm 09(330)$ & 0.522 & .13 \\
\hline Positive cough, no. (\%) & $1132(99)$ & $911(99)$ & $228(98)$ & 0.504 & .32 \\
\hline Positive gag, no. (\%) & $806(93)$ & $654(94)$ & $152(89)$ & 0.521 & .06 \\
\hline Positive corneal, no. (\%) & $889(97)$ & $722(98)$ & $167(97)$ & 0.503 & .55 \\
\hline Pupil size, mm & $2.8 \pm 0.8(1,683)$ & $2.8 \pm 0.8(1,359)$ & $2.8 \pm 0.7(324)$ & 0.508 & .96 \\
\hline Pupil reaction, no. (\%) & $1440(85)$ & $1165(85)$ & $275(85)$ & 0.508 & .51 \\
\hline Admission SAPS II & $39 \pm 15(2,007)$ & $38 \pm 15(1,631)$ & $44 \pm 16(376)$ & 0.615 & $<.01$ \\
\hline \multicolumn{6}{|c|}{$\begin{array}{l} \pm \text { Values are mean } \pm \mathrm{SD} \text { (number of observations). } \\
\text { * Area under the receiver operating characteristic curve from bivariate logistic regression. } \\
\dagger \text { Logistic regression } P \text { with robust standard errors. } \\
\text { SAPS = Simplified Acute Physiology Score }\end{array}$} \\
\hline
\end{tabular}

statistically significant. We used statistics software for the analyses (Stata 11.0, StataCorp, College Station, Texas). Data are presented as mean $\pm \mathrm{SD}$ unless otherwise noted.

\section{Results}

Data from 2,007 subjects were analyzed, of whom 379 (19\%) failed extubation at any time during the hospital stay and required out-of-operating room reintubation. The most common reason for reintubation was respiratory failure $(n=287,76 \%)$, followed by airway obstruction $(n=65$, $17 \%$ ). Early reintubation (within $24 \mathrm{~h}$ ) was observed in $155(7.7 \%)$ subjects. Details of the study population have been previously reported. ${ }^{5}$ Overall, the subjects were $49.5 \pm 17.9$ years of age, $66 \%$ were male, and the mean SAPS II score was $39.4 \pm 15.2$. Subjects who required reintubation any time during their ICU or hospital stay tended to be older and have a higher mean SAPS II. Type of admission or admitting service (general surgery, orthopedics, vascular, neurosurgery, head and neck, medicine, $P=.64)$, presence of traumatic injury $(P=.87)$, and body mass index were not associated with failed extubation. The prevalence of acute lung injury or acute respiratory dis- tress syndrome at any time during hospitalization was similar between the groups.

The respiratory, airway, cardiovascular, and neurological variables measured prior to initiating the SBT are displayed in Table 1, stratified by extubation failure at any time. Some respiratory and cardiovascular variables and measures of secretion burden differed between the 2 groups. None of the neurological exams, such as Glasgow coma score, pupil diameter and reactivity, or presence of cough/ gag reflex, differed significantly between the groups.

Respiratory and cardiovascular variables during the SBTs are shown in Table 2. The predictors of extubation failure during the SBTs were largely respiratory variables (see Table 2). Variables measured within 1 hour of extubation are shown in Table 3. Lower $\mathrm{S}_{\mathrm{pO}_{2}}$ was associated with extubation failure.

In the multivariate analysis, independent predictors associated with reintubation at any time during hospitalization were higher admission SAPS II score, higher secretion burden (either suctioning frequency or cumulative amount of secretions in $24 \mathrm{~h}$ ), higher minute ventilation (either immediately prior to or during the SBT), higher number of SBTs prior to extubation, and lower diastolic 
Table 2. Characteristics During the Spontaneous Breathing Trials

\begin{tabular}{|c|c|c|c|c|c|}
\hline & $\begin{array}{l}\text { All Patients } \\
(n=2,007)\end{array}$ & $\begin{array}{l}\text { Not Reintubated } \\
\quad(n=1,628)\end{array}$ & $\begin{array}{l}\text { Reintubated } \\
(n=379)\end{array}$ & AUC* & $P \dagger$ \\
\hline $\begin{array}{l}\text { Number of prior spontaneous } \\
\text { breathing trials }\end{array}$ & $2.6 \pm 2.3(1,706)$ & $2.4 \pm 2.0(1,374)$ & $3.5 \pm 3.0(332)$ & 0.636 & $<.01$ \\
\hline Tidal volume, $\mathrm{mL}$ & $533 \pm 99(1,793)$ & $534 \pm 97(1,477)$ & $526 \pm 108(316)$ & 0.523 & .15 \\
\hline Breathing frequency, breaths/min & $18 \pm 6(1,971)$ & $18 \pm 6(1,597)$ & $20 \pm 7(374)$ & 0.605 & $<.01$ \\
\hline Minute ventilation, $\mathrm{L} / \mathrm{min}$ & $7.7 \pm 3.8(1,890)$ & $7.5 \pm 3.7(1,539)$ & $8.4 \pm 4.1(351)$ & 0.563 & $<.01$ \\
\hline $\begin{array}{l}\text { Rapid shallow breathing index } \\
\text { (breathing frequency/tidal volume) }\end{array}$ & $36 \pm 29(1,785)$ & $35 \pm 24(1,471)$ & $41 \pm 45(314)$ & 0.597 & .31 \\
\hline $\mathrm{PEEP}, \mathrm{cm} \mathrm{H}_{2} \mathrm{O}$ & $5.1 \pm 0.8(1,825)$ & $5.1 \pm 0.8(1,479)$ & $5.1 \pm 0.7(346)$ & 0.502 & .59 \\
\hline $\mathrm{F}_{\mathrm{IO}_{2}}$ & $0.41 \pm 0.11(1,894)$ & $0.41 \pm 0.11(1,540)$ & $0.41 \pm 0.10(354)$ & 0.496 & .67 \\
\hline $\mathrm{S}_{\mathrm{pO}_{2}}, \%$ & $99 \pm 2(1,971)$ & $99 \pm 2(1,600)$ & $98 \pm 2(374)$ & 0.563 & $<.01$ \\
\hline $\mathrm{pH}$ & $7.41 \pm 0.05(1,842)$ & $7.41 \pm 0.05(1,496)$ & $7.41 \pm 0.05(346)$ & 0.499 & .47 \\
\hline $\mathrm{P}_{\mathrm{aCO}_{2}}, \mathrm{~mm} \mathrm{Hg}$ & $41 \pm 7(1,842)$ & $41 \pm 7(1,496)$ & $41 \pm 7(346)$ & 0.511 & .64 \\
\hline $\mathrm{P}_{\mathrm{aO}_{2}}, \mathrm{~mm} \mathrm{Hg}$ & $141 \pm 55(1,842)$ & $144 \pm 56(1,496)$ & $126 \pm 45(346)$ & 0.600 & $<.01$ \\
\hline $\mathrm{P}_{\mathrm{aO}_{2}} / \mathrm{F}_{\mathrm{IO}_{2}}, \mathrm{~mm} \mathrm{Hg}$ & $357 \pm 796(1,783)$ & $371 \pm 882(1,447)$ & $300 \pm 110(340)$ & 0.599 & $<.01$ \\
\hline Heart rate, beats/min & $93 \pm 19(1,971)$ & $93 \pm 19(1,600)$ & $94 \pm 20(371)$ & 0.514 & .39 \\
\hline Systolic blood pressure, $\mathrm{mm} \mathrm{Hg}$ & $132 \pm 22(1,971)$ & $133 \pm 22(1,600)$ & $131 \pm 24(371)$ & 0.524 & .26 \\
\hline Intracranial pressure, $\mathrm{cm}_{2} \mathrm{O}$ & $9.8 \pm 6.4(190)$ & $10.2 \pm 6.3(141)$ & $8.6 \pm 6.9(49)$ & 0.556 & .10 \\
\hline
\end{tabular}

Table 3. Data From the First 60 Minutes After Extubation

\begin{tabular}{|c|c|c|c|c|c|}
\hline & $\begin{array}{l}\text { All Patients } \\
(n=2,007)\end{array}$ & $\begin{array}{l}\text { Not Reintubated } \\
\quad(n=1,628)\end{array}$ & $\begin{array}{l}\text { Reintubated } \\
(n=379)\end{array}$ & AUC* & $P \dagger$ \\
\hline $\mathrm{S}_{\mathrm{pO}_{2}}, \%$ & $98 \pm 3(1,971)$ & $98 \pm 3(1,600)$ & $97 \pm 5(371)$ & 0.557 & $<.01$ \\
\hline Heart rate, beats/min & $96 \pm 19(1,971)$ & $96 \pm 19(1,600)$ & $98 \pm 20(371)$ & 0.525 & .07 \\
\hline Systolic blood pressure, $\mathrm{mm} \mathrm{Hg}$ & $134 \pm 23(1,969)$ & $134 \pm 22(1,599)$ & $136 \pm 25(371)$ & 0.520 & .19 \\
\hline Intracranial pressure, $\mathrm{cm} \mathrm{H}_{2} \mathrm{O}$ & $9.2 \pm 6.2(164)$ & $9.3 \pm 6.6(120)$ & $9.0 \pm 4.7(44)$ & 0.487 & .77 \\
\hline
\end{tabular}

blood pressure prior to the SBT (Table 4). Independent predictors of the need for reintubation within 24 hours were higher admission SAPS II score, lower oxygenation (either $\mathrm{P}_{\mathrm{aO}_{2}} / \mathrm{F}_{\mathrm{IO}_{2}}$ or $\mathrm{P}_{\mathrm{aO}_{2}}$ from the SBT arterial blood analysis, or $\mathrm{S}_{\mathrm{pO}_{2}}$ during the SBT), and higher secretion burden (either suctioning frequency or cumulative amount of secretions in $24 \mathrm{~h}$, see Table 4 ). When determining sensitivity and specificity, both multivariate models showed approximately $70 \%$ accuracy in correctly predicting whether an individual subject would fail extubation during the pre-specified time period. Figures 1 and 2 show the receiver operating characteristic curves.

\section{Discussion}

The decision to discontinue invasive mechanical ventilation involves weighing the benefits of avoiding the mor- bidity associated with prolonged mechanical ventilation against the risk of morbidity from extubation failure. ${ }^{10}$ Individual risk factors associated with extubation failure have been previously reported, ${ }^{11}$ but studies reporting clinically useful decision tools are sparse. Herein we report the generation of 2 prediction models for extubation failure in subjects who have passed an SBT: one for failure at any time during hospitalization, and another for failure in the first $24 \mathrm{~h}$ after extubation. The models only retained 4 (early failure) and 5 (failure at any time) clinically accessible variables, respectively, that were highly contributory to predict extubation failure. It is noteworthy that despite some overlap (disease severity, minute ventilation, and secretion burden), the variables predicting early failure were not the same as those predicting failure at any time. Oxygenation was an important component of early failure, 
Table 4. Multivariable Logistic Regression Model for Extubation Failure

\begin{tabular}{|c|c|c|c|c|c|}
\hline & Odds Ratio & $95 \% \mathrm{CI}$ & AUC* & $95 \% \mathrm{CI}$ & $P$ \\
\hline Failure at any time after extubation & & & 0.68 & $0.65-0.72$ & \\
\hline SAPS II & 1.02 & $1.01-1.03$ & & & $<.01$ \\
\hline Suctioning frequency & 1.05 & $1.02-1.09$ & & & $<.01$ \\
\hline Number of prior SBTs & 1.12 & $1.06-1.19$ & & & $<.01$ \\
\hline Diastolic blood pressure & 0.99 & $0.98-1.00$ & & & .01 \\
\hline Minute ventilation during SBT & 1.03 & $1.00-1.07$ & & & .06 \\
\hline Failure within 24 hours of extubation & & & 0.72 & $0.68-0.75$ & \\
\hline SAPS II & 1.01 & $1.00-1.02$ & & & $<.01$ \\
\hline Suctioning frequency & 1.10 & $1.06-1.14$ & & & $<.01$ \\
\hline $\mathrm{S}_{\mathrm{pO}_{2}}$ & 0.91 & $0.84-0.98$ & & & .01 \\
\hline Minute ventilation during SBT & 1.04 & $0.99-1.08$ & & & .10 \\
\hline
\end{tabular}

* Area under the receiver operating characteristic curve from bivariate logistic regression.

SAPS $=$ Simplified Acute Physiology Score

$\mathrm{SBT}=$ spontaneous breathing trial

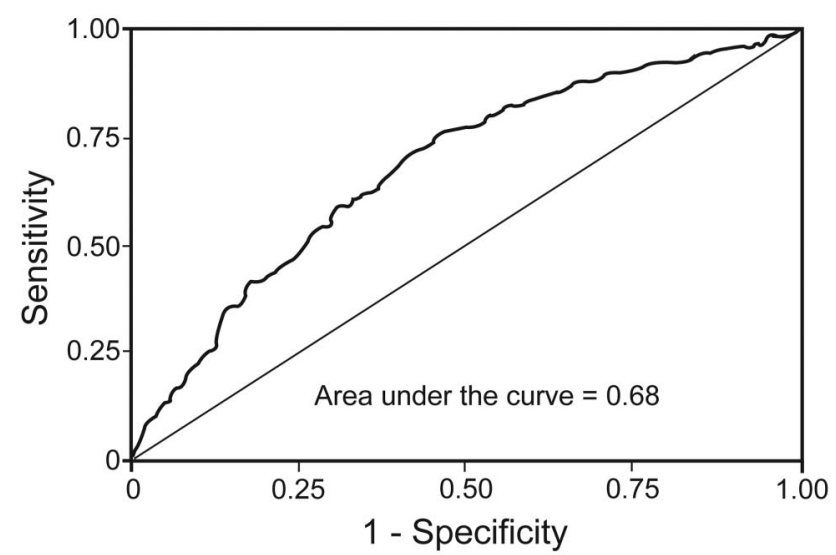

Fig. 1. Receiver operating characteristic curve from the multivariable logistic regression model, with robust variance estimation, for the prediction of extubation failure at any time during the hospital stay. The variables included in the model were Simplified Acute Physiology Score (SAPS) II, number of spontaneous breathing trials (SBTs) prior to extubation, diastolic blood pressure prior to SBT, frequency of tracheal suctioning in the previous 24 hours, and minute ventilation during the SBT. Equation: Logit $[\mathrm{YX}]=$ $-2.24+0.019 \times$ SAPS II score $+0.113 \times$ number of prior SBTs $-0.012 \times$ diastolic blood pressure $+0.049 \times$ suctioning frequency $+0.034 \times$ minute ventilation. The probability of extubation failure at any time $=$ expit $(\operatorname{logit}[Y X])$, where expit $(x)=e^{x} /\left(1+e^{x}\right)$.

whereas lower diastolic pressure and repeatedly failed SBT were significant contributors to failure at any time.

Both models had an area under the receiver operating characteristic curve of approximately $70 \%$, indicating fairly good accuracy. ${ }^{12}$ Although this accuracy might be increased by the addition of more variables, this would be at the expense of including covariates with minor contribution to explain the model variability, and adding complexity to the data gathering by the clinician. Adding variables with small contribution to overall variability could

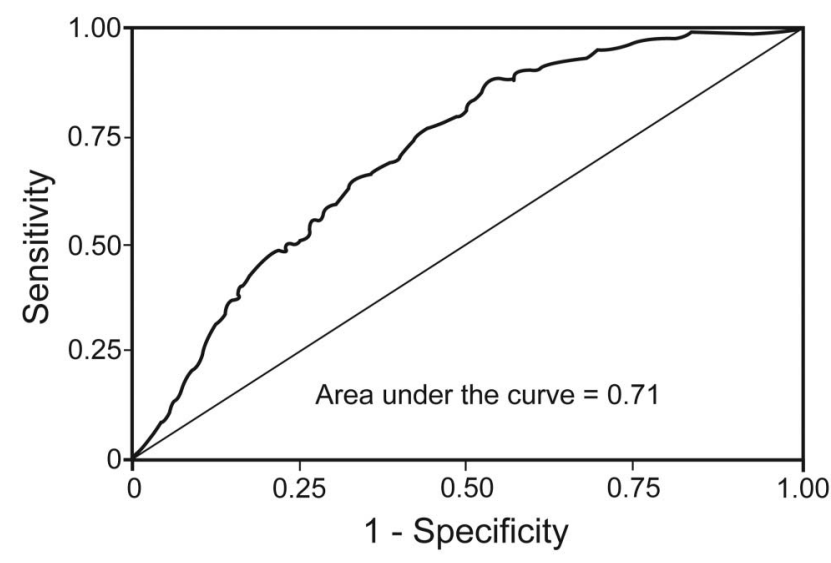

Fig. 2. Receiver operating characteristic curve from the multivariable logistic regression model, with robust variance estimation, for the prediction of extubation failure within 24 hours of extubation. The variables included in the model were Simplified Acute Physiology Score (SAPS) II, $\mathrm{S}_{\mathrm{pO}_{2}}$ during the SBT, frequency of tracheal suctioning in the previous $24 \mathrm{~h}$, and minute ventilation during the SBT. Equation: Logit $[\mathrm{YX}]=10.07+0.015 \times$ SAPS II score $-0.140 \times$ oxygenation $+0.108 \times$ suctioning frequency $+0.035 \times$ minute ventilation. Probability of extubation failure within 24 hours $=$ expit (logit $[Y X])$, where expit $(x)=e^{x} /\left(1+e^{x}\right)$.

also affect the model performance, due to overfitting resulting in reduced generalizability to other settings.

In both models, SAPS II independently predicted extubation failure, which is consistent with 2 prior studies.9,13 Similar to previous reports, ${ }^{7}$ we found that increased secretion burden was a significant contributor to extubation failure, in both models. It seems intuitive that patients who cannot adequately perform pulmonary toilet are at greater risk for upper-airway obstruction, inspissated secretions and resultant dependent post-obstructive atelectasis, increased elastic and resistive work of breathing, and elevated intrapulmonary shunt fraction. Indeed, $\mathrm{P}_{\mathrm{aO}} / \mathrm{F}_{\mathrm{IO}_{2}}$, 
which provides a rough approximation of shunt fraction, ${ }^{14}$ was also a significant predictor in the early extubation failure model. Further, other investigators have reported that the absolute amount of tracheal secretions and inadequate cough response increases the risk of extubation failure in patients who no longer appear to need mechanical ventilation. ${ }^{6,15,16}$ Consistent with a previous report, ${ }^{11}$ another risk factor for extubation failure was the number of previously failed SBTs, which suggests that, despite a pattern of improvement over time, some patients with more difficult weaning may not have achieved complete resolution of acute respiratory failure and may be more likely to require reintubation. ${ }^{17}$ Frutos-Vivar et al also reported that positive fluid balance in the previous 24 hours was a significant predictor of extubation failure. ${ }^{11}$ However, the predictive ability of this variable, even when combined with rapid shallow breathing index, was weak. This finding might be related to the variability of fluid balance over the ICU course and the accuracy of the recording of the balance in the medical record. A recent study at our institution examined fluid balance in patients with subarachnoid hemorrhage, and suggested extensive day-to-day variability in the response to fluid administration. ${ }^{18}$

We defined early extubation failure as reintubation within 24 hours, as the median time to reintubation was 22 hours, ${ }^{5}$ thereby capturing more than half of extubation failures within this window. Furthermore, 24 hours is an important post-extubation milestone in the decision to transfer a patient out of the ICU after liberation from mechanical ventilation.

For extubation failure at any time we found lower diastolic blood pressure a significant risk factor for extubation failure. This has not been reported previously. Different explanations to interpret this finding can be proposed, including cardiac-related weaning failure or autonomic dysfunction. Recently, transthoracic echocardiography during SBT documented impaired ventricular relaxation and diastolic dysfunction, leading to cardiac-related weaning failure. ${ }^{19}$ Spontaneous inspiratory efforts can precipitate leftventricular dilatation and cardiogenic shock in patients without coronary artery disease, ${ }^{20}$ and lower arterial diastolic pressure may reflect spontaneous-ventilation-induced decrease in intrathoracic pressure. ${ }^{21}$ However, lower diastolic blood pressure could also be a surrogate of autonomic nervous system dysfunction and reflect increased efferent sympathetic nerve activity.

Neurologic function, as assessed by Glasgow coma score, was not associated with extubation failure in either model. In a cohort of 122 subjects mechanically ventilated for $\geq 48$ hours, Mokhlesi and colleagues reported a Glasgow coma score $<10$ to confer a 13 -fold increase in the risk of extubation failure. ${ }^{7}$ In a randomized controlled trial comparing a non-physician-directed ventilator weaning protocol to routine care in a neurosurgical ICU, Namen et al reported a Glasgow coma score $\geq 8$ to be strongly associated with extubation success in neurosurgical patients. ${ }^{22}$ Salam and colleagues reported that subjects unable to complete 4 task commands (open eyes, follow with eyes, grasp hand, and stick out tongue) prior to extubation were 4 times more likely to require reintubation. ${ }^{6}$ However, the study by Coplin et al reported similar extubation failure among brain injured subjects regardless of their Glasgow coma score. ${ }^{23}$ In addition, using the Full Outline of UnResponsiveness (FOUR) score, which combines assessment of eyes, brainstem, reflexes, and respiration, to assess mental status in 62 subjects with a primary neurologic injury, Ko and colleagues reported similar scores among those who were successfully extubated and those that failed. ${ }^{24}$ In all likelihood, it is the combination of mental status and the ability to clear the upper and lower airways that determines successful extubation in patients who have passed an SBT. The SBT reflects the patient's ability to maintain adequate oxygenation and ventilation without, or with minimal, support. It is that crucial second aspect of successful extubation- the complete removal of positive pressure and the necessity of the artificial airway-that remains in question. ${ }^{17}$ Our data support the greater importance of respiratory secretions over mentation.

The present study is one of the largest to date, both in the number of subjects and the number of variables examined, on predictors of extubation failure in the ICU. Because we captured data over a 15 -month period, historical trend should not influence the results. Due to our large sample size, the differences in some variables were statistically significant but not necessarily clinically relevant. Small absolute differences in continuous variables were contributory when jointly combined with other variables in the model and weighted based on their regression coefficient. The joint effect of all weighted variables included in the model yielded the individual predicted probabilities of extubation failure.

Several limitations of our study are worth noting. The data collection was retrospective, so data not recorded were not available. Subjective assessments, such as the grading of the quality and quantity of secretions, were not standardized. And the decision to extubate rested with the primary critical care service, and primary specialty, level of training, and practice patterns may have differed among the physicians. Given that all the subjects were in only one medical center, the generalizability of our results to patients in other regions and to hospitals with different practice styles may be limited.

\section{Conclusions}

In summary, we have identified a few important risk factors for extubation failure at any time during hospitalization and within the first 24 hours of extubation. These 


\section{Predictors of Reintubation in CRitically Ill Patients}

independent variables explained a substantial portion of the variability in extubation failure, and can help identify patients at high risk of failure. Under both models, disease severity, secretion burden, and minute ventilation were significant predictors of extubation failure. Additionally, lower oxygenation was a risk factor for failure within 24 hours, whereas the number of previously failed SBTs and lower diastolic pressure were risk factors for failure at any time.

These data imply that, once a patient is deemed ready for extubation, the clinician can identify patients who require more post-extubation vigilance, and devise a backup plan that might include continuation of high-intensity monitoring, prompt availability of supplies for reintubation, or request an immediate consultation by respiratory therapists to initiate aggressive bronchial hygiene protocols. Future studies are needed to prospectively validate and optimize this prediction model by capturing additional potentially important variables that may play a role in extubation failure, such as response to fluid administration or biomarkers of disease (troponin, brain natriuretic peptide, and pro-brain natriuretic peptide).

\section{REFERENCES}

1. Ely EW, Baker AM, Dunagan DP, Burke HL, Smith AC, Kelly PT, et al. Effect on the duration of mechanical ventilation of identifying patients capable of breathing spontaneously. N Engl J Med 1996; 335(25):1864-1869.

2. Yang KL, Tobin MJ. A prospective study of indexes predicting the outcome of trials of weaning from mechanical ventilation. N Engl J Med 1991;324(21):1445-1450.

3. Liu Y, Wei LQ, Li GQ, Lv FY, Wang H, Zhang YH, et al. A decision-tree model for predicting extubation outcome in elderly patients after a successful spontaneous breathing trial. Anesth Analg 2010;111(5):1211-1218.

4. Su WL, Chen YH, Chen CW, Yang SH, Su CL, Perng WC, et al. Involuntary cough strength and extubation outcomes for patients in an ICU. Chest 2010;137(4):777-782.

5. Menon N, Joffe AM, Deem S, Yanez ND, Grabinsky A, Dagal AH, et al. Occurrence and complications of tracheal reintubation in critically ill adults. Respir Care 2012;57(10):1555-1563.

6. Salam A, Tilluckdharry L, Amoateng-Adjepong Y, Manthous CA. Neurologic status, cough, secretions and extubation outcomes. Intensive Care Med 2004;30(7):1334-1339.

7. Mokhlesi B, Tulaimat A, Gluckman TJ, Wang Y, Evans AT, Corbridge TC. Predicting extubation failure after successful completion of a spontaneous breathing trial. Respir Care 2007;52(12):1710-1717.
8. Girard TD, Kress JP, Fuchs BD, Thomason JW, Schweickert WD, Pun BT, et al. Efficacy and safety of a paired sedation and ventilator weaning protocol for mechanically ventilated patients in intensive care (Awakening and Breathing Controlled trial): a randomised controlled trial. Lancet 2008;371(9607):126-134.

9. Esteban A, Alía I, Gordo F, Fernandez R, Solsona JF, Vallverdú I, et al. Extubation outcome after spontaneous breathing trials with T-tube or pressure support ventilation. The Spanish Lung Failure Collaborative Group. Am J Respir Crit 1997; Care Med 156(2 Pt 1): 459-465.

10. Thille AW, Harrois A, Schortgen F, Brun-Buisson C, Brochard L. Outcomes of extubation failure in medical intensive care unit patients. Crit Care Med 2011;39(12):2612-2618.

11. Frutos-Vivar F, Ferguson ND, Esteban A, Epstein SK, Arabi Y, Apezteguía C, et al. Risk factors for extubation failure in patients following a successful spontaneous breathing trial. Chest 2006;130(6): 1664-1671.

12. Mandrekar JN. Receiver operating characteristic curve in diagnostic test assessment. J Thorac Oncol 2010;5(9):1315-1316.

13. Epstein SK, Ciubotaru RL, Wong JB. Effect of failed extubation on the outcome of mechanical ventilation. Chest 1997;112(1):186-192.

14. Covelli HD, Nessan VJ, Tuttle WK 3rd. Oxygen derived variables in acute respiratory failure. Crit Care Med 1983;11(8):646-649.

15. Smina M, Salam A, Khamiees M, Gada P, Amoateng-Adjepong Y, Manthous CA. Cough peak flows and extubation outcomes. Chest 2003;124(1):262-268.

16. Khamiees M, Raju P, DeGirolamo A, Amoateng-Adjepong Y, Manthous CA. Predictors of extubation outcome in patients who have successfully completed a spontaneous breathing trial. Chest 2001;120(4):1262-1270.

17. Tobin MJ. Extubation and the myth of "minimal ventilator settings." Am J Respir Crit Care Med 2012;185(4):349-350.

18. Martini RP, Deem S, Brown M, Souter MJ, Yanez ND, Daniel S, et al. The association between fluid balance and outcomes after subarachnoid hemorrhage. Neurocrit Care 2012;17(2):191-198.

19. Caille V, Amiel JB, Charron C, Belliard G, Vieillard-Baron A, Vignon P. Echocardiography: a help in the weaning process. Crit Care 2010;14(3):R120.

20. Buda AJ, Pinsky MR, Ingels NB Jr, Daughters GT 2nd, Stinson EB, Alderman EL. Effect of intrathoracic pressure on left ventricular performance. N Engl J Med 1979;301(9):453-459.

21. Pinsky MR. Breathing as exercise: the cardiovascular response to weaning from mechanical ventilation. Intensive Care Med 2000; 26(9):1164-1166.

22. Namen AM, Ely EW, Tatter SB, Case LD, Lucia MA, Smith A, et al. Predictors of successful extubation in neurosurgical patients. Am J Respir Crit Care Med 2001;163(3 Pt 1):658-664.

23. Coplin WM, Pierson DJ, Cooley KD, Newell DW, Rubenfeld GD. Implications of extubation delay in brain-injured patients meeting standard weaning criteria. Am J Respir Crit Care Med 2000;161(5): 1530-1536

24. Ko R, Ramos L, Chalela JA. Conventional weaning parameters do not predict extubation failure in neurocritical care patients. Neurocrit Care 2009;10(3):269-273.

This article is approved for Continuing Respiratory Care Education credit. For information and to obtain your CRCE

(free to AARC members) visit

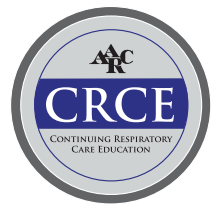

www.rcjournal.com 